\title{
Perspective rectification of integral images produced using arrays of circular lenses
}

\author{
E. T. Koufogiannis, ${ }^{*}$ N. P. Sgouros, and M. S. Sangriotis \\ Department of Informatics and Telecommunications, University of Athens Panepistimiopolis, Athens 15784, Greece \\ *Corresponding author: efthimis@di.uoa.gr
}

Received 5 April 2013; revised 6 June 2013; accepted 6 June 2013;

posted 7 June 2013 (Doc. ID 188373); published 9 July 2013

\begin{abstract}
There are many different three-dimensional (3D) techniques to capture and deliver autostereoscopic 3D content. A promising technique that provides two-dimensional parallax as well as high-quality, fullcolor 3D content is integral imaging (InI). Misalignments between the lens arrays (LAs) and the camera charged coupled device, however, introduce geometric distortions in the acquired image that propagate through the different image processing stages and deteriorate the 3D effect. Here, we propose a method to accurately rectify the perspective distortion of integral images (InIms) generated using circular lenses. Using an edge-linking approach, we extracted elliptically shaped contours of elemental images in the perspectively distorted InIm. To calculate the rectification matrix, we used the images of the circular points. Subsequently, we applied a triangulation scheme followed by a statistical approach to accurately estimate the grid structure of the LA. Finally, we provided experimental results over a wide range of InIms to evaluate the robustness and accuracy of the proposed method using objective metrics. (C) 2013 Optical Society of America

OCIS codes: (080.0080) Geometric optics; (100.6890) Three-dimensional image processing; (100.5010) Pattern recognition; (110.2990) Image formation theory; (110.3010) Image reconstruction techniques.

http://dx.doi.org/10.1364/AO.52.004959
\end{abstract}

\section{Introduction}

Integral photography (IP) was introduced by the Nobel Laureate Lippman [1] in 1908. Now, integral imaging (InI) is based on the concept of IP and is considered one of the most successful techniques to acquire and display three-dimensional (3D) images and video [2]. InI makes possible the delivery of accurately detailed, full-color $3 \mathrm{D}$ content with support for multiple simultaneous users.

Figure 1(a) shows the basic InI acquisition setup, which consists of a charged coupled device (CCD) and a lens array (LA) [3, $\underline{4}$. Figure 1(b) shows the display setup, which consists of a liquid crystal display (LCD) and a LA.

$1559-128 \mathrm{X} / 13 / 204959-10 \$ 15.00 / 0$

(C) 2013 Optical Society of America
When placing an appropriate LA between the CCD and the scene, each of the lenses comprising the LA forms an elemental image (EI) on the CCD sensor, which is a partial micrography of the acquired scene. The acquired set of EIs forms an integral image (InIm), which can be used to reproduce the 3D scene using an LCD and appropriate Las, as already described.

LAs currently used in existing InI setups are categorized according to the shape of the used lenses. Lenses can have different regular shapes, and Fig. $\underline{2}$ depicts several such LAs containing circular, square, hexagonal, and triangular lenses.

In this work, we address the issue of perspective rectification of InIms acquired using LAs with circular lenses, which are widely used in existing setups. In addition, circular LAs are easier to manufacture and offer better optical properties because of their intrinsic spherical shape. Thus, a number of already 


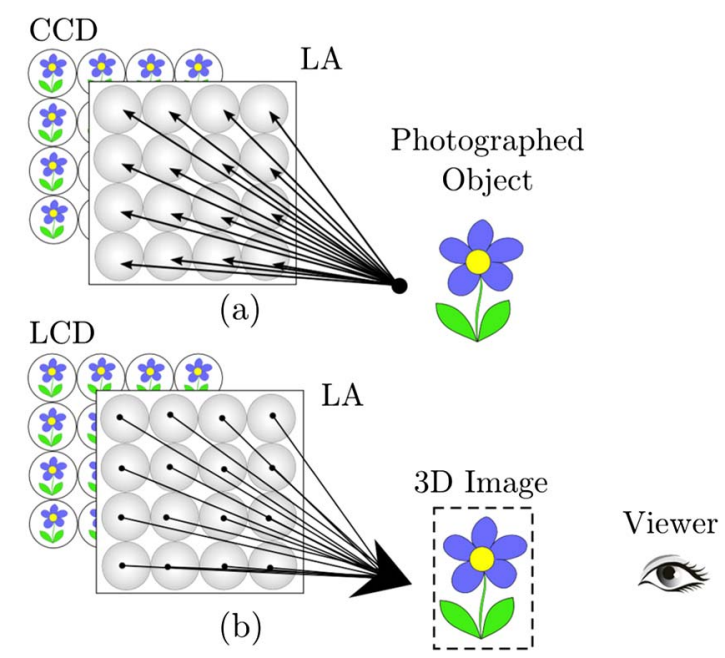

Fig. 1. (a) InI acquisition and (b) InI display setup.

existing applications like microscopy [5] could benefit from an automated method to detect misalignments when using LAs with circular lenses.

In detail, the most important concern during the acquisition procedure should be the accurate alignment between the CCD and LA planes. Due to misalignment between the two planes, perspective distortion may be introduced, which damages the shapes and causes variability in the size of the acquired EIs.

Therefore, EIs are perspectively distorted into ellipses in the case of circular lenses and irregular polygons in the case of square, hexagonal, or triangular lenses. The result is poor $3 \mathrm{D}$ representation through an InI display, deformation of the 3D object reconstructed after using the procedure described in [6] or a distorted, single-view 2D subimage after applying the method described in [7]. Furthermore, the majority of InIm coding algorithms convert InIms in EI sequences and require accurate knowledge of the proper geometry of the InIm structure [8]. Clearly, a rectification process is required to alleviate these geometric aberrations. Figure 3 illustrates the effect of perspective distortion on the InIm acquired using a LA with circular lenses.

Since perspective distortion cannot be easily avoided without significant integration costs, various software methods have been proposed to eliminate this distortion. Until now, these methods worked with InI setups containing square, hexagonal, or triangular lenses and used an edge detector to extract line segments of the acquired InIm. Length and angle measurements on these segments are used as input to the metric rectification procedure of [9] to calculate the perspective rectification parameters.

A method for the rectification of square lens InIms is presented in [10]. This method uses the Hough transform to detect a rectangle of the distorted EI grid, which is subsequently used to estimate the rectification parameters. An alternative rectification approach for square LA InIms is proposed in [11] and offers a robust grid estimation method with strong statistical support for line segments over the entire InIm. The method proposed in [12] is a generalization of [11] for the case of hexagonal LAs.

The above frameworks consist of a preprocessing stage to accurately determine the underlying image structures (EI borders), a method to calculate a set of parameters that will be used in the rectification process matrices and the application of the rectification matrices on the distorted image. However, such approaches fail to deal accurately with LAs consisting of circular lenses as they use straight line-fitting techniques to approximate EI borders.

Here, we propose a methodology that retains the final optimized process for applying the rectification matrices and provides a robust method that uses the

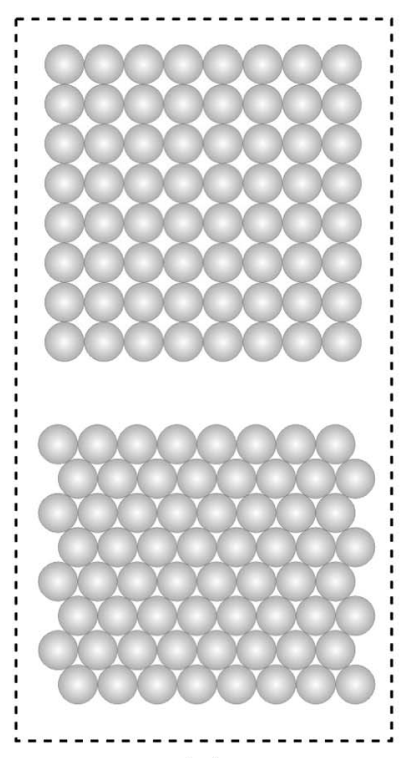

(a)

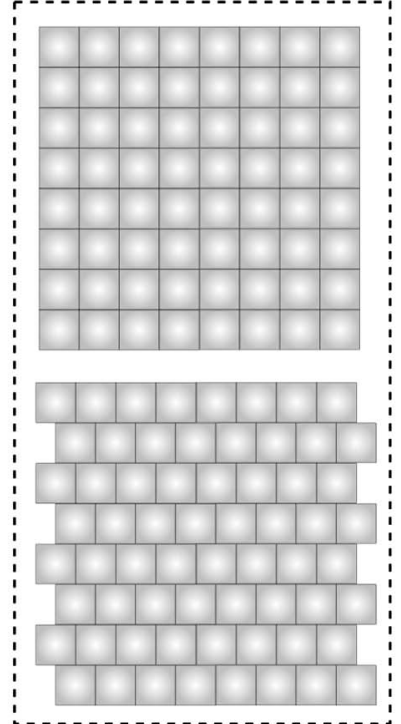

(b)

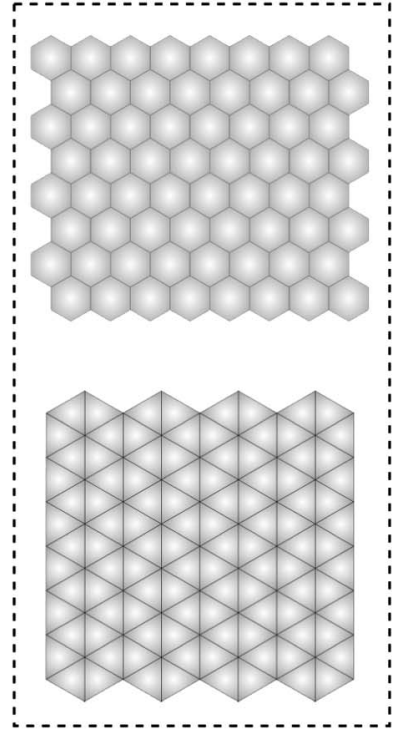

(c)

Fig. 2. Various LAs consisting of (a) circular, (b) square, and (c) hexagonal and triangular lenses. 


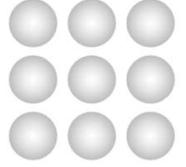

(a)

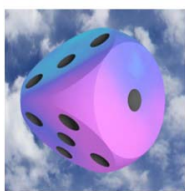

(c)
Perspective

Distortion
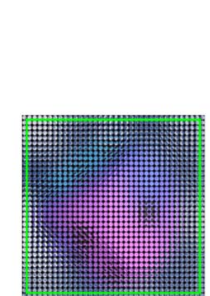

(d) (b)

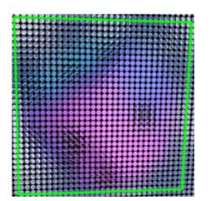

(e)
Fig. 3. (a) Undistorted coplanar circular EIs, (b) perspectively distorted EIs, (c) a conventional photograph of a 3D dice, (d) acquired InIm of the dice without distortion, and (e) perspectively distorted acquired InIm. The borders in (d) and (e) are shown for illustration purposes.

unique properties of circular lenses to perform the preprocessing step. We also developed and applied an analytically derived methodology to map the resulting elliptical shapes to parameters required to calculate the rectification matrices.

Our paper and its key components are arranged as follows:

- Section 2 presents the necessary mathematical background of this work. More analytically, in Section 2.A, we describe the parameters of the required planar rectification matrices.

- Section 2.B shows how these parameters can be calculated when the only available information is elliptical contours instead of line segments as in the case of LAs with circular lenses.

- Section 2.C provides a method to fit ellipses to points, which is necessary to estimate analytic equations of ellipses contained in an acquired InIm.

- Section 3 explains how we applied these mathematical principles in a real-world scenario. Sections 3.A and 3.B estimate the ellipse equations of EI contours in a distorted InIm. In Section 3.C we used these ellipses to calculate the rectification matrices using a statistical approach.

- Section 4 contains the evaluation methodology as well as the experimental setup. In Section 4.A, we describe the experimental InIm datasets and the pickup process, while Sections 4.B and 4.C contain the description of the geometric consistency metrics as well as the evaluation of the images in the experimental InIm datasets.

- Section $\underline{5}$ summarizes the most important outcomes and contributions of our work. Explicit mathematical details and proofs related to the equations of Section 2.B are provided in Appendix A.

\section{Mathematical Background}

\section{A. Perspective Rectification Matrices}

As described in [ $[\underline{9}, \underline{10}]$, and [11], a point $P$ of a perspectively distorted plane is mapped to its corresponding point $P^{\prime}$ on the rectified (geometrically corrected) plane using

$$
\vec{x}^{\prime}=H \vec{x},
$$

where $\vec{x}, \vec{x}^{\prime}$ are 3 -vectors representing the homogeneous coordinates of $P, P^{\prime}$, and $H$ is the $3 \times 3$ real-valued perspective rectification matrix. Furthermore, the perspective transformation $H$ can be written as the product of three $3 \times 3$ real-valued matrices according to the equation:

$$
H=H_{s} H_{a} H_{p} .
$$

Matrices $H_{p}, H_{a}, H_{s}$ represent a pure perspective, an affine, and a similarity transformation, respectively, and are given by:

$$
\begin{aligned}
& H_{p}=\left(\begin{array}{ccc}
1 & 0 & 0 \\
0 & 1 & 0 \\
l_{1} & l_{2} & l_{3}
\end{array}\right), \\
& H_{a}=\left(\begin{array}{ccc}
\frac{1}{\beta} & -\frac{\alpha}{\beta} & 0 \\
0 & 1 & 0 \\
0 & 0 & 1
\end{array}\right) \\
& H_{s}=\left(\begin{array}{ccc}
R & x_{0} \\
& & y_{0} \\
0 & 0 & 1
\end{array}\right)\left(\begin{array}{ccc}
c & 0 & 0 \\
0 & c & 0 \\
0 & 0 & 1
\end{array}\right),
\end{aligned}
$$

and

$$
R=\left(\begin{array}{cc}
\cos \theta & \sin \theta \\
-\sin \theta & \cos \theta
\end{array}\right),
$$

where $l_{1}, l_{2}, l_{3}$ are the parameters defining the distorted plane's vanishing line [9], $\alpha, \beta$ are the metric correction parameters, and $H_{s}$ results in rotation by $R$, translation by $\left(x_{0}, y_{0}\right)^{T}$ and scaling by $c$.

To rectify a perspectively distorted plane, we calculated the required parameters for $H_{p}, H_{a}, H_{s}$. The application of $H_{p}, H_{a}, H_{s}$ for rectification purposes is used regardless of the EI geometries contained inside the InIm plane [13]. However, based on the EI geometries, different approaches are followed to derive these matrices. In the case of EIs with circular geometries, the distorted InIm plane contains coplanar ellipses corresponding to circles on the

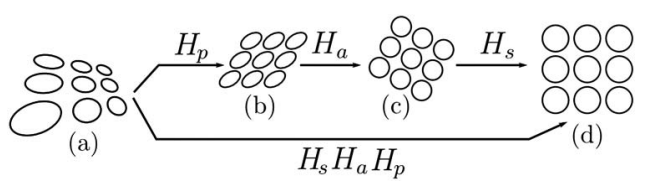

Fig. 4. (a) Set of coplanar ellipses corresponding to perspectively distorted circles, (b) the affine plane of ellipses resulting after applying $H_{p}$, (c) the metric-corrected ellipses transformed to circles after applying $H_{a}$, and (d) the final and correctly rotated and scaled set of coplanar circles after applying the similarity transformation $H_{s}$. 
undistorted plane, as shown in Fig. 3(b). The sequential application of the matrices $H_{p}, \overline{H_{a}}, H_{s}$ shown in Fig. 4 demonstrates the stages recovering the geometric properties of perspectively distorted plane consisting of ellipses.

\section{B. Rectification Using the Images of the Circular Points}

We calculate the parameters that compose $H_{p}, H_{a}$, $H_{s}$ to perform planar rectification. In square, hexagonal, and other lens geometries detected straight line segments are used to produce lines that are finally used for parameter estimation [9].

However in our case, straight lines are unavailable. Perspective distortion in InIms acquired from LAs with circular lenses makes the acquired EIs have an elliptical contour. In this case, line detection methods are of no use due to the complete lack of straight edges between the EIs.

To this end, we followed an approach based on the acquired ellipses of the plane to calculate the required rectification parameters. The central part of all ellipse-based rectification methods is the estimation of the images of the circular points (ICPs) $[14,15]$.

In the proposed framework, we used the approach of ICPs to to extract the parameters $l_{1}, l_{2}, l_{3}, \alpha, \beta$ to perform metric rectification on the acquired InIm.

The circular points (CPs), which lie on the undistorted plane, are the two complex conjugate 3 -vectors $(1, \pm i, 0)^{T}[13]$ and are located at infinity on the vanishing line. Furthermore, it is easy to notice that they verify the generic homogeneous equation of a circle with radius $r$ and center $(m, n, 1)$ :

$$
(X-m Z)^{2}+(Y-n Z)^{2}=r^{2} Z^{2} .
$$

This means that any possible pair of circles in the undistorted plane verifies these points. Additionally, the matrix form of a generic conic $C$ is given by:

$$
C=\left(\begin{array}{ccc}
a & b / 2 & d / 2 \\
b / 2 & c & e / 2 \\
d / 2 & e / 2 & f
\end{array}\right) .
$$

According to [13], the conic $C$ is mapped under a perspective transformation $H$ to a corresponding conic $C^{\prime}$ using the equation:

$$
H^{-T} C H^{-1}=C^{\prime} .
$$

Figure 5 shows that under the perspective distortion $H^{-1}$, circles are mapped to ellipses and the two conjugate CPs are mapped to two conjugate ICPs that have the form [15]:

$$
\operatorname{ICPs}=\left(\alpha l_{3} \mp i l_{3} \beta, l_{3},-l_{2}-l_{1} \alpha \pm i l_{1} \beta\right)^{T} .
$$

From Eq. (10), we notice that ICPs involve all required rectification parameters contained in transformation matrices $H_{p}, H_{a}$.

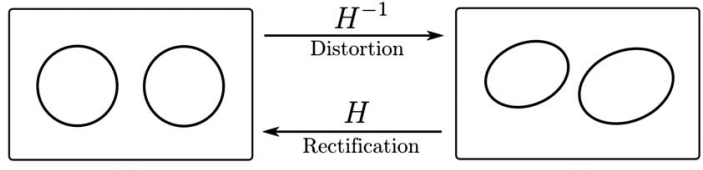

(a)

(c)

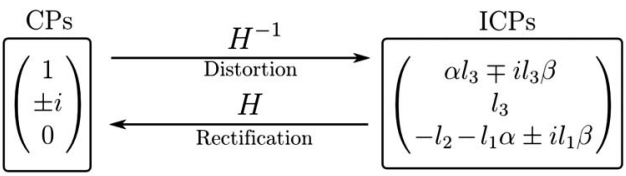

(b)

(d)

Fig. 5. (a) Any pair of coplanar circles verifies the CPs shown in (b) under the perspective distortion $H^{-1}$, the pair of ellipses in (c) corresponding to the circles in (a) verifies the ICPs shown in (d).

ICPs are estimated by finding the complex solutions verifying a pair of ellipses on the distorted plane. The analytic equation of a $2 \mathrm{D}$ ellipse is

$$
a x^{2}+b x y+c y^{2}+d x+e y+f=0,
$$

therefore, two ellipses form the system:

$$
\left\{\begin{array}{l}
a_{1} x^{2}+b_{1} x y+c_{1} y^{2}+d_{1} x+e_{1} y+f_{1}=0 \\
a_{2} x^{2}+b_{2} x y+c_{2} y^{2}+d_{2} x+e_{2} y+f_{2}=0
\end{array} .\right.
$$

A method for solving this quadratic system has been proposed in [14]. In our case, two ellipses do not visually intersect on the acquired image plane; therefore, the four solutions $\left(x_{s}, y_{s}\right)$ contain only complex coordinates. These come in two conjugate pairs:

$$
\left\{\begin{array}{ll}
\left(x_{s 0}, y_{s 0}\right), & \left(\overline{x_{s 0}}, \overline{y_{s 0}}\right) \\
\left(x_{s 1}, y_{s 1}\right), & \left(\overline{x_{s 1}}, \overline{y_{s 1}}\right)
\end{array} .\right.
$$

Correctly selecting the two ICPs from the previous four solutions relies on the fact that the ICPs verify the equation of every ellipse. Solving two or more pairs of ellipses makes ICPs estimation a matter of locating the shared solutions, or in the case of noise, the closest ones in terms of proximity on the complex $x y$-plane [14].

After we identify the ICPs by solving systems of ellipse pairs, their estimated values are $\mathrm{ICP}_{1}=$ $\left(x_{c}, y_{c}\right)$ and $\operatorname{ICP}_{2}=\left(\overline{x_{c}}, \overline{y_{c}}\right)$. Since the ICPs lie on the plane's vanishing line $\mathbf{I}=\left(l_{1}, l_{2}, l_{3}\right)^{T}$, the parameters $l_{1}, l_{2}, l_{3}$ are calculated as the cross product of the two ICP homogeneous vectors [15]:

$$
\left(l_{1}, l_{2}, l_{3}\right)^{T}=\left(x_{c}, y_{c}, 1\right)^{T}=\left(\overline{x_{c}}, \overline{y_{c}}, 1\right)^{T} .
$$

The metric rectification parameters $\alpha, \beta$ are subsequently given by:

$$
\left\{\begin{array}{l}
\alpha=\operatorname{Real}\left(\frac{-l_{2} x_{c}}{l_{3}+l_{1} x_{c}}\right)=\operatorname{Real}\left(\frac{l_{3}+l_{2} y_{c}}{-l_{1} y_{c}}\right) \\
\beta=\left|\operatorname{Imag}\left(\frac{-l_{2} x_{c}}{l_{3}+l_{1} x_{c}}\right)\right|=\left|\operatorname{Imag}\left(\frac{l_{3}+l_{2} y_{c}}{-l_{1} y_{c}}\right)\right|
\end{array}\right.
$$


An analytic proof of the previous equation as well as the detailed transformation of the CPs using the perspective distortion matrix $H^{-1}$ is given in Appendix $\underline{\text { A. }}$.

\section{Ellipse Parameter Fitting}

Estimating the analytic parameters of ellipses in the acquired distorted plane is the first important step toward rectification. Therefore, we propose a method of fitting ellipses to lists of contiguous $2 \mathrm{D}$ points using the approach that follows.

Let $S=\left\{\left(x_{i}, y_{i}\right)\right\}, i=1 \ldots M$ a sequence of $M$ discrete image points belonging to a single elliptically shaped contour. We will estimate the analytic parameters of the ellipse $C$ passing from all the points in $S$. The generic 2D ellipse equation is given by Eq. (11). Since this ellipse must verify all of the points $\left(x_{i}, y_{i}\right) \in S$, the posed problem can be formulated in vector form as the homogeneous overdetermined linear system:

$$
A \mathbf{u}=\mathbf{0},
$$

where

$$
A=\left(\begin{array}{cccccc}
x_{1}^{2} & x_{1} y_{1} & y_{1}^{2} & x_{1} & y_{1} & 1 \\
x_{1}^{2} & x_{2} y_{2} & y_{2}^{2} & x_{2} & y_{2} & 1 \\
\vdots & \vdots & \vdots & \vdots & \vdots & \\
x_{M}^{2} & x_{M} y_{M} & y_{M}^{2} & x_{M} & y_{M} & 1
\end{array}\right)
$$

and

$$
\mathbf{u}=(a, b, c, d, e, f)^{T} .
$$

The vector $\mathbf{u}$ contains the six unknown ellipse parameters and the $M \times 6$ matrix $A$ is evaluated using all of the points $\left(x_{i}, y_{i}\right), i=1 \ldots . M$. It is evident that due to noise, Eq. (16) does not have an exact zero solution; therefore we seek the best approximation to it: that is, the $\mathbf{u}$ that minimizes $\|A \mathbf{u}\|$.

Since the solution vector $\mathbf{u}$ can be arbitrarily scaled, a reasonable constraint would be to find solution for which $\|\mathbf{u}\|=1$. The minimization of $\|A \mathbf{u}\|$ subject to the constraint $\|\mathbf{u}\|=1$ can be performed in a least squares sense by applying singular value decomposition (SVD) on the matrix A:

$$
A=U D V^{T} .
$$

The estimate solution $\hat{\mathbf{u}}$ is the last column of the matrix $V$ and contains the fitted ellipse parameters. The mean square error (MSE) for all the points contained in the sequence $S$ is given by

$$
\operatorname{MSE}=\frac{1}{M}\|A \hat{\mathbf{u}}\| .
$$

\section{Application to the Real Case}

In this section, we present the details of the ellipsebased rectification theory to robustly rectify acquired InIms where the presence of noise is inevitable. We also describe the technique used for ellipse extraction and fitting as well as the approach we followed to estimate the rectification matrix parameters.

\section{A. Elliptical Edge Extraction}

The initial step of our framework is the extraction of elliptically shaped EI edges from the acquired InIm. We converted the acquired and perspectively distorted InIm shown in Fig. 6(a) to its grayscale version and applied an optimal thresholding algorithm [16] that results in a binary image with separated EI texture and lens border background.

To extract the elliptical lens border edges, we employed a boundary isolation technique [17] that resulted in an edge image containing elliptical lens border edges along with some random shapes corresponding to the random texture of the InIm content.

\section{B. Edge Linking and Ellipse Fitting}

After generating the edge image, we used an edgelinking function to collect edges in lists containing pixel coordinates.

Various image processing libraries offer this functionality such as [18] and [19]. Edge-linking functions operate on a binary image, collect connected pixels that represent edges and assign them to lists of points. In this work, we used the framework implemented in [19] to register $N$ edges and store the

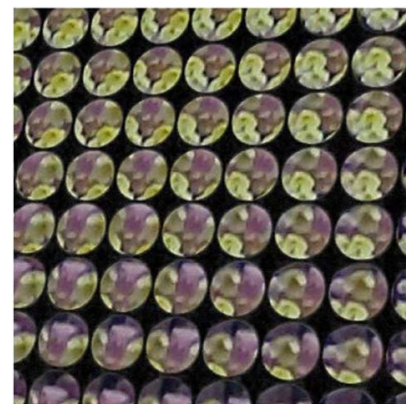

(a)

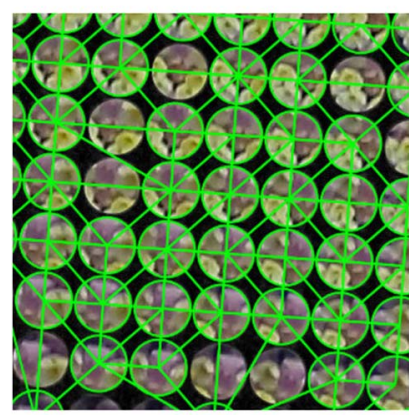

(c)

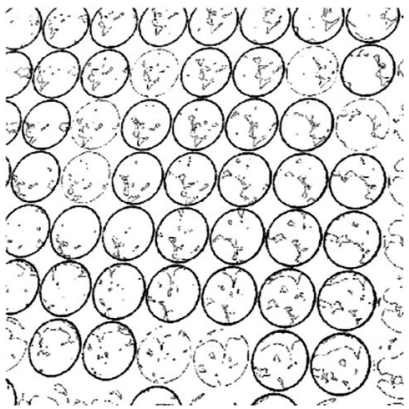

(b)

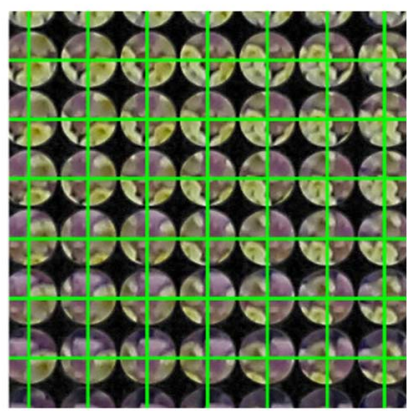

(d)
Fig. 6. (a) Distorted acquired InIm, (b) edge image with fitted ellipses overlaid, (c) rectified image and Delaunay triangulation on the rectified circle centers, and (d) correctly rotated image with the registered grid superimposed. 
corresponding pixels in the sequences $S_{i}, i=1 \ldots N$. Each of these sequences contains a variable number of contiguous $2 \mathrm{D}$ points.

Subsequently we used the fitting model described in Section 2.C for each sequence $S_{i}$ to fit an ellipse $C_{i}$ and calculated the corresponding fit error $\operatorname{MSE}(i)$. We collected and sorted these errors in ascending order. Finally, we kept the ellipses corresponding to the smallest $20 \%$ of the sorted error population, which ensured that point lists that gave inaccurate fits were rejected. The resulting fitted ellipses overlaid on the edge image are shown in Fig. 6(b). The fitted ellipses are subsequently used to calculate the rectification parameters using the approach that follows.

\section{Estimation of Rectification Parameters}

The resulting ellipse equations correspond to elliptical lens borders in the distorted InIm. We formed all possible ellipse pairs $\left(C_{i}, C_{j}\right)$ and solved the system formed by Eq. (12). To discard possible degeneracies, we accepted only systems generating two pairs of conjugate solutions. These solutions have the form of Eq. (13), as seen in Section 2.B. and are collected in the sequence:

$$
P=\left\{\left(x_{s 0}, y_{s 0}\right),\left(\overline{x_{s 0}}, \overline{y_{0}}\right)\left(x_{s 1}, y_{s 1}\right),\left(\overline{x_{s 1}}, \overline{y_{s 1}}\right), \ldots\right\} .
$$

The points contained in sequence $P$ that correspond to the ICPs would ideally have exactly the same coordinates and they would generate the same vanishing line using Eq. (14). However, the existence of noise induces large deviations from the ideal case.

To this end, we followed a simple approach to identify the ICPs: For each conjugate solution pair stored in $P$, we calculated the line $\mathbf{I}_{i}$ given by the crossproduct:

$$
\mathbf{I}_{i}=\left(x_{s i}, y_{s i}\right)^{T} \times\left(\overline{x_{s i}}, \overline{y_{s i}}\right)^{T} .
$$

For each $\mathbf{I}_{i}$, we estimated the distance from the image origin and generated the corresponding sequence $d_{i}$. Figure 7 shows the histogram of $d_{i}$. The correct ICP candidates produced a vanishing line that does not intersect the distorted image and is located outside the image area. Furthermore, since the correct ICPs coordinates have some perturbation instead of being identical for every combination of $\left(C_{i}, C_{j}\right)$, they produced a lobe in the histogram of $d_{i}$.

We isolated the lobe shown in Fig. 7 and subsequently located the solutions corresponding to it.

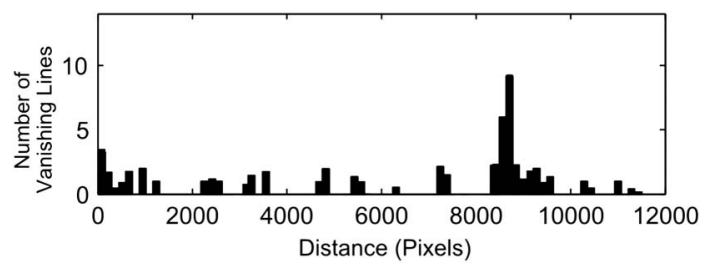

Fig. 7. Histogram of candidate vanishing line distances from the image origin. The lobe corresponds to correct ICPs.
The ICPs $\left(x_{c}, y_{c}\right),\left(\overline{x_{c}}, \overline{y_{c}}\right)$ are calculated as the median of the isolated solutions.

Using Eqs. (14), (15), we calculated the vanishing line parameters $l_{1}, \bar{l}_{2}, l_{3}$ that produced matrix $H_{p}$ as well as the metric rectification parameters $\alpha, \beta$ which produced matrix $H_{a}$. We subsequently applied $H_{p}$ and $H_{a}$, resulting in a slightly rotated image with all metric properties corrected as shown in Fig. 6(c). The registered ellipses $C_{i}$ are transformed to the corresponding circles $C_{i^{\prime}}$ using Eq. (9).

We subsequently applied Delaunay triangulation on the resulting circle centers $\left\{\left(c x_{i}, c y_{i}\right)\right\}$, which resulted in the line segment sequence $\left\{\mathrm{LS}_{i}\right\}$. We also calculated the angle sequence $\left\{\theta_{i}\right\}$ which contains the angles formed by each segment in $\left\{\mathrm{LS}_{i}\right\}$ and the $x$ axis and produced its histogram shown in Fig. 8 . Next we isolated the intervals $\left[-10^{\circ}, 10^{\circ}\right]$ and $\left[80^{\circ}\right.$, $100^{\circ}$ ] and locate their corresponding maximum values $\theta_{1}, \theta_{2}$. The segments corresponding to a radius of $1^{\circ}$ around $\theta_{1}, \theta_{2}$ are isolated and form two new segment populations denoted as $\left\{S_{h}\right\},\left\{S_{v}\right\}$. These contained segments that are approximately vertical. We calculated the values:

$$
x_{h}=\frac{\operatorname{mean}\left(\Delta x\left\{S_{h}\right\}\right)+\operatorname{mean}\left(\Delta y\left\{S_{v}\right\}\right)}{2},
$$

and

$$
y_{h}=\frac{\operatorname{mean}\left(\Delta y\left\{S_{h}\right\}\right)+\operatorname{mean}\left(\Delta x\left\{S_{v}\right\}\right)}{2},
$$

and the rotation matrix of Eq. (ㅁ) as:

$$
R=-\frac{1}{\sqrt{x_{h}^{2}+y_{h}^{2}}}\left(\begin{array}{cc}
x_{h} & y_{h} \\
-y_{h} & x_{h}
\end{array}\right) \text {. }
$$

After applying $R$, we used the method described in [11] to fit accurate grid lines on the segments that connected the EI centers. The EI lens pitch in pixels was set as the mean distance between the estimated grid lines.

\section{Experiments and Results}

\section{A. InIm Datasets}

The effectiveness of the proposed method was assessed over a large number $(K=30)$ of optically acquired InIms that were purposely captured with perspective distortion. The experimental setup used the technique described in [10]. For the pickup assembly, we used a Canon EOS-500D camera in

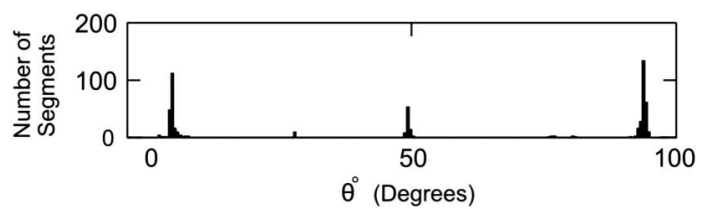

Fig. 8. Angle histogram of the segments connecting the circle centers after rectification. 
conjunction with a LA with circular lenses. During pickup, we introduced slight perspective misalignment to produce the distorted InIms. Note that for the purposes of this work, lens distortions are not considered because the camera firmware automatically compensates for basic barrel and pincushion distortions. In addition to the InIm set of real-world scenes, we generated a large number of perspectively distorted raytraced InIms $(L=50)$ containing different gradients, colors, shapes, textures, and EI sizes using the method described in [20]. The artificial InIms were further contaminated with various levels of Gaussian noise to assess the overall robustness of our framework under variable noise conditions.

\section{B. Evaluation Criteria}

To evaluate the rectification performance of the proposed framework, we generated the distortion matrices for the raytraced InIms using Eqs. (3)-(6) by setting the parameters $l_{1}, l_{2}, \alpha, \beta, \theta$ to $a$ priori known values. We omitted the parameter $l_{3}$, since the vanishing line is represented in normalized form [13]. The rectification parameters for all the raytraced InIms were subsequently estimated with respect to their preset ground truth values along with the corresponding mean error rate percentages.

We also used two additional objective metrics that statistically characterized the geometrical consistency of the InIm after applying the proposed rectification framework.

For this purpose, we used the fact that the ideal square grid formed by the registered grid on the InIm shown in Fig. 6 in an undistorted InIm contains equally spaced intersecting segments forming angles of $90^{\circ}$. To evaluate deviations from this ideal grid that characterize the undistorted InIm, we calculated the angles $\{\omega\}$ formed between all the intersecting grid segments and the normalized grid segments lengths $\{\lambda\}$. Normalization of the line segments was performed to provide cumulative results of experiments over InIms with different EI sizes. Finally, using $\{\omega\},\{\lambda\}$ we calculated $\overline{\omega_{s}}$ and $\overline{\lambda_{s}}$ for each dataset and their respective standard deviations $\sigma_{\omega_{s}}$ and $\sigma_{\lambda_{s}}$. This was done to evaluate deviations with respect to the undistorted InIms in the utilized datasets.

Next we summarized the evaluation of our framework's rectification results on the InIm sets of the raytraced as well as the optically acquired images. Figure $\underline{9}$ displays a representative image from each set showing the $2 \mathrm{D}$ image of the scene, the acquired InIms, and their rectified counterparts.

Figure 10 shows the $2 \mathrm{D}$ side view rendered subimages [7], using the undistorted, the distorted, and the rectified InIms that resulted after applying our rectification framework as shown in Fig. 9 .

Finally we derived the plots shown in Fig. 11 by using the InIm of Fig. 9(c) contaminated with various levels of Gaussian noise as input to our algorithm. Using the formulas in [8], we evaluated the output PSNR of the rectified EIs shown in Fig. 9(e). In Fig. 11, we plotted the mean value PSNR as well as the variance represented by the standard deviation $\sigma$ PSNR.

\section{Results}

Table 1 summarizes the rectification parameters mean relative error for the entire raytraced InIm dataset. As this table shows, the relative error for all involved rectification parameters remains below $3 \%$ regardless of the noise levels and the InIm-specific characteristics (texture and scene setup).

Table 2 summarizes the overall geometric consistency values along with their standard deviations for the entire raytraced InIm set. As this table shows, the standard deviation of the cumulative error in $\overline{\omega_{s}}$ is well below $1^{\circ}$ even in highly noise contaminated InIms. In addition, $\overline{\lambda_{s}}$ is under $0.04(4 \%)$ regardless of the noise level.

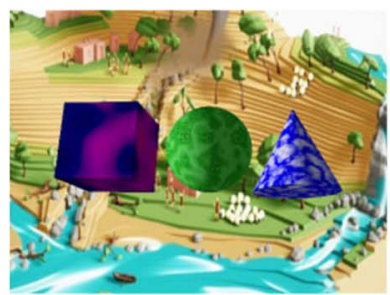

(a)

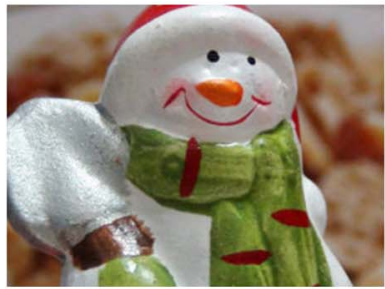

(b)

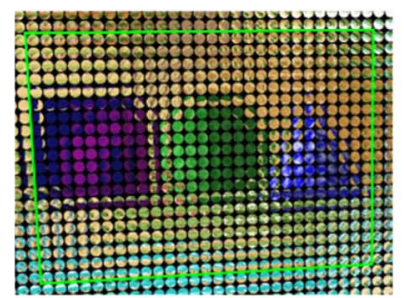

(c)

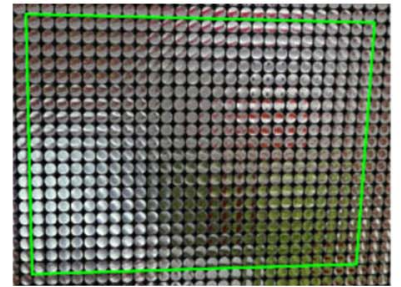

(d)

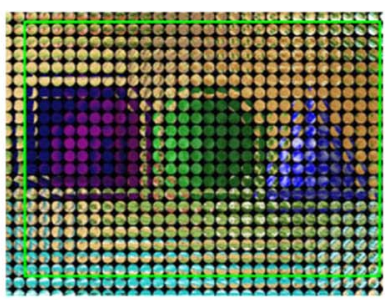

(e)

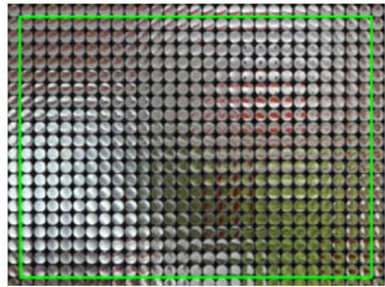

(f)

Fig. 9. Rectification on a raytraced and an optically acquired image: (a) A "3D Objects" scene, (b) an optically acquired "Toy," (c),(d) the corresponding acquired and distorted InIms, and (e),(f) rectified InIms using the proposed method. The borders in (c),(f) are shown for illustration purposes. 


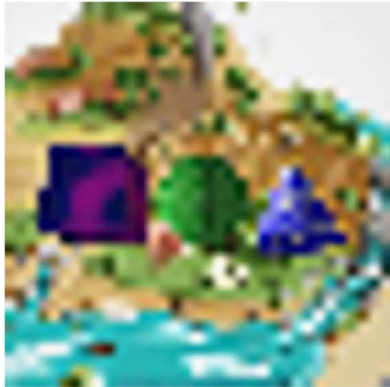

(a)

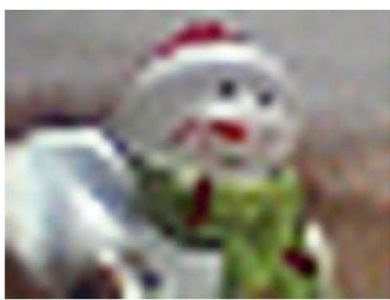

(b)

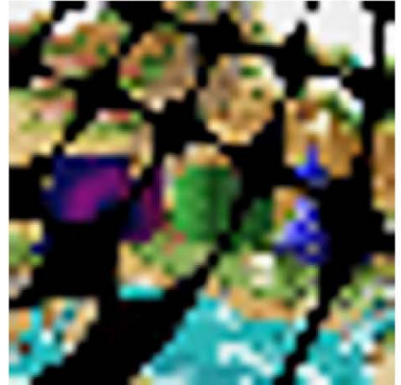

(c)

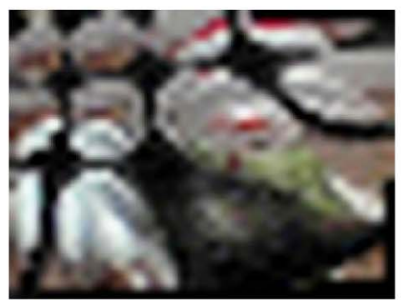

(d)

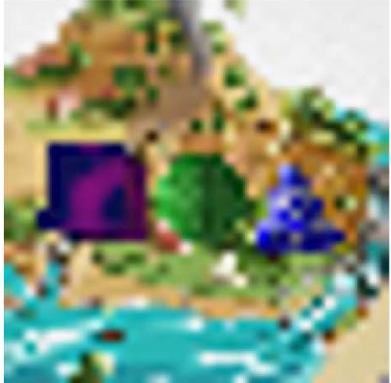

(e)

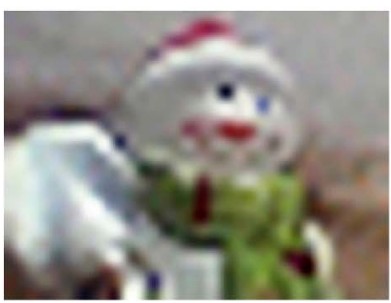

(f)

Fig. 10. (a),(b) Side view subimages rendered from the undistorted "3D Objects" and "Toy" InIms, (c),(d) side view subimages of the

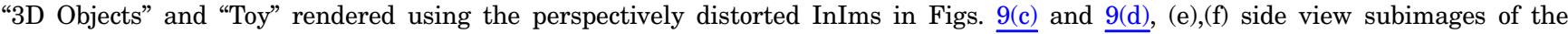
"3D Objects" and "Toy" rendered using the rectified InIms of Figs. $\underline{9(\mathrm{e})}$ and $\underline{9(\mathrm{f})}$.
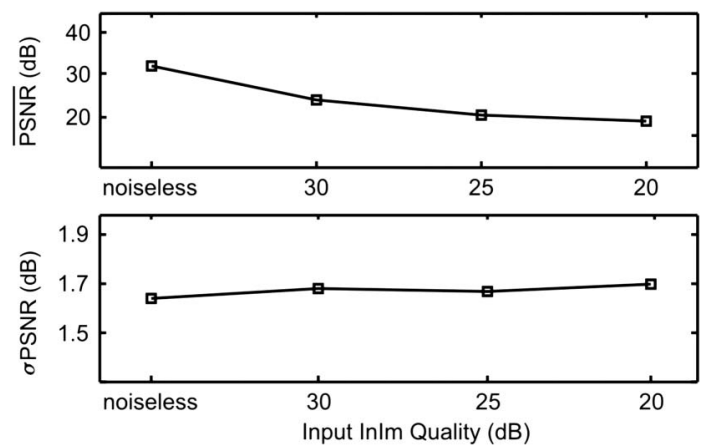

Fig. 11. Mean value and standard deviation of the PSNR sequence for the rectified EIs of Fig. 9(c). The horizontal axis denotes the input InIm quality, while the vertical axis denotes the evaluated PSNR sequence of the EIs in the geometrically corrected InIm.

Tables 3 and 4 summarize the respective results for the InIm displayed in the first row of Fig 9 , which is included in the set, representing the scene " $3 \mathrm{D} \mathrm{Ob-}$ jects" with textured objects in a complex background.

The side view subimage renderings corresponding to Fig. 9 are shown in Fig 10 and the corresponding rectified EIs PSNR sequence is evaluated in the plots of Fig. 11 with respect to various noise levels of the

Table 1. Mean Relative Error Percentages of the Rectification Parameters for the Entire Raytraced InIm Set

\begin{tabular}{lccccc}
\hline & $l_{1}(\%)$ & $l_{2}(\%)$ & $\alpha(\%)$ & $\beta(\%)$ & $\theta(\%)$ \\
\hline Noiseless $(\mathrm{dB})$ & 0.55 & 0.71 & 0.72 & 0.81 & 0.65 \\
30 & 0.96 & 1.21 & 0.85 & 0.94 & 0.66 \\
25 & 1.29 & 1.58 & 1.13 & 1.03 & 1.12 \\
20 & 2.02 & 2.06 & 1.79 & 1.77 & 1.09 \\
\hline
\end{tabular}

input InIm. The plots shown in Fig. 11 verify that the proposed InIm rectification approach retains a high performance ratio even when applied on heavily noise contaminated InIms.

Additionally, we have summarized the results for the geometrical consistency parameters over the optically acquired InIm dataset in Table $\underline{5}$ and the

Table 2. Cumulative Geometric Consistency Results for the Raytraced Inim Set

\begin{tabular}{lcc}
\hline & $\overline{\omega_{s}} \pm \sigma_{\omega_{s}}$ & $\sigma_{\lambda_{s}}$ \\
\hline Noiseless $(\mathrm{dB})$ & $90.00 \pm 0.09^{\circ}$ & 0.010 \\
30 & $90.00 \pm 0.13^{\circ}$ & 0.013 \\
25 & $90.00 \pm 0.44^{\circ}$ & 0.021 \\
20 & $90.00 \pm 0.76^{\circ}$ & 0.034 \\
\hline
\end{tabular}

Table 3. Relative Error Percentages of the Rectification Parameters for "3D Objects"

\begin{tabular}{lccccc}
\hline & $l_{1}(\%)$ & $l_{2}(\%)$ & $\alpha(\%)$ & $\beta(\%)$ & $\theta(\%)$ \\
\hline Noiseless $(\mathrm{dB})$ & 0.67 & 0.92 & 0.93 & 1.05 & 0.76 \\
30 & 1.03 & 1.12 & 1.01 & 1.27 & 1.16 \\
25 & 1.17 & 1.25 & 1.32 & 1.64 & 1.87 \\
20 & 1.74 & 1.53 & 1.94 & 2.16 & 2.29 \\
\hline
\end{tabular}

Table 4. Geometric Consistency Results for "3D Objects"

\begin{tabular}{lcc}
\hline & $\overline{\omega_{i}}+\sigma_{\omega_{i}}$ & $\sigma_{\lambda_{i}}$ \\
\hline Noiseless $(\mathrm{dB})$ & $90.00 \pm 0.15^{\circ}$ & 0.014 \\
30 & $90.00 \pm 0.17^{\circ}$ & 0.022 \\
25 & $90.00 \pm 0.52^{\circ}$ & 0.037 \\
20 & $90.00 \pm 0.87^{\circ}$ & 0.041 \\
\hline
\end{tabular}


Table 5. Cumulative Geometric Consistency Results for All InIms of the Optically Acquired Set

\begin{tabular}{lc}
\hline$\overline{\omega_{i}}+\sigma_{\omega_{i}}$ & $\sigma_{\lambda_{s}}$ \\
\hline $90.00 \pm 0.38^{\circ}$ & 0.043 \\
\hline
\end{tabular}

Table 6. Geometric Consistency Results for the "Toy"

\begin{tabular}{cc}
\hline$\overline{\omega_{i}}+\sigma_{\omega_{i}}$ & $\sigma_{\lambda_{i}}$ \\
\hline $90.00 \pm 0.46^{\circ}$ & 0.039 \\
\hline
\end{tabular}

scene "Toy" displayed in the second row of Fig. 9 in Table 6 . The data in Tables 5 and 6 show that both measured angle deviation and segment length are low, remaining well below $0.5^{\circ}$ and $0.05(5 \%)$ respectively, in all InIms in the dataset.

\section{Conclusions}

In this work, we presented a detection and rectification framework for perspective distortion that affects InIms acquired using arrays with circular lenses. For this purpose, we derived analytic formulas to calculate the parameters of the rectification matrices and established an automated process to directly estimate these parameters from raw image data. The proposed method is characterized by increased robustness using data from multiple non-intersecting detected ellipses. To this end, we proposed a novel way to correctly select the ICPs to minimize the total error introduced during the preprocessing stages.

We finally assessed the robustness and accuracy of the technique by estimating the relative error in the calculation of the rectification parameters and measuring the geometric consistency of the rectified InIm using objective quality metrics. Furthermore, the side view subimages shown in Fig. 10 as well as the InIm PSNR evaluation of Fig. 11 verify the high performance and robustness of the proposed framework to restore the properties of perspectively distorted InIms. In the evaluation process, we used large datasets of optically acquired as well as raytraced InIms to examine the parameters that may affect the robustness of the rectification framework. Note that the computationally generated InIm set allowed joint control of both perspective distortion and noise levels.

As already presented in the respective tables, the geometric consistency is retained in the InIms of both sets and is slowly varying for different noise levels. Since noise greatly affected the rectification framework at the early processing stages and resulted in large propagation errors we concluded that the proposed framework is robust against high noise contamination levels.

Note that the ellipse-based fitting and rectification approach is specifically designed to treat cases with circular lenses; however, it also could be applied to other lens geometries. In these cases, the geometric and perspective centers of the distorted shapes do not coincide [15], which yields multiple ellipses that fit a specific distorted rectangle. Hence, this approach will raise the uncertainty in the preprocessing and parameter calculation stages for other lens geometries and finally reduce the overall accuracy of the rectification process. On the contrary, it is more convenient to integrate the proposed method in already proposed frameworks to treat circular-lensbased geometries.

This research has been cofinanced by the European Union (European Social Fund, or ESF) and Greek national funds through the Operational Program, "Education and Lifelong Learning," of the National Strategic Reference Framework (NSRF) Research Funding Program: Heracleitus II, "Investing in knowledge society through the European Social Fund."

\section{Appendix A: ICPs Parameter Estimation}

The CPs are invariant to the similarity transformation $H_{s}$ [13]. Therefore, they are mapped to their corresponding ICPs under the perspective distortion matrix $\left(H_{a} H_{p}\right)^{-1}[\underline{15}]$ using the following equation:

$$
\left(H_{a} H_{p}\right)^{-1}(1, \pm i, 0)^{T}=\mathrm{ICP}_{1,2} .
$$

After performing the required multiplication and inversion of the matrices $H_{p}, H_{a}$ that are analytically given in Eqs. (3), (ㅁ) we derive the ICPs final form:

$$
\mathrm{ICP}_{1,2}=\left(\alpha l_{3} \mp i l_{3} \beta, l_{3},-l_{2}, l_{1} \alpha \pm i l_{1} \beta\right)^{T} .
$$

The ICPs are calculated by solving multiple systems of ellipse equation pairs on the distorted image plane. Therefore, their numeric values are given by:

$$
\operatorname{ICP}_{1}=\left(x_{c}, y_{c}\right), \quad \operatorname{ICP}_{2}=\left(\overline{x_{c}}, \overline{y_{c}}\right) .
$$

The vanishing line's parameters $l_{1}, l_{2}, l_{3}$ are calculated using $x_{c}, y_{c}$ :

$$
\left(l_{1}, l_{2}, l_{3}\right)^{T}=\left(x_{c}, y_{c}, 1\right)^{T} \times\left(\overline{x_{c}}, \overline{y_{c}}, 1\right)^{T} .
$$

Now since $l_{1}, l_{2}, l_{3}, x_{c}, y_{c}$ have been estimated, the plane's metric rectification parameters $\alpha, \beta$ are calculated as follows:

We homogenize Eq. (A2), which yields

$$
\left\{\begin{array}{l}
\left(x_{c}, y_{c}, 1\right)=\left(\frac{\alpha l_{3}-i l_{3} \beta}{-l_{2}-l_{1} \alpha+i l_{1} \beta}, \frac{l_{3}}{-l_{2}-l_{1} \alpha+i l_{1} \beta}, 1\right) \\
\left(\overline{x_{c}}, \overline{y_{c}}, 1\right)=\left(\frac{\alpha l_{3}+i l_{3} \beta}{-l_{2}-l_{1} \alpha-i l_{1} \beta}, \frac{l_{3}}{-l_{2}-l_{1} \alpha+i l_{1} \beta}, 1\right)
\end{array},\right.
$$

and after simplifying and removing the redundant conjugate equations, it results in:

$$
\alpha-i \beta=\frac{-l_{2} x_{c}}{l_{3}+l_{1} x_{c}}=\frac{l_{3}+l_{2} y_{c}}{-l_{1} y_{c}},
$$


and

$$
\left\{\begin{array}{l}
\alpha=\operatorname{Real}\left(\frac{-l_{2} x_{c}}{l_{3}+l_{1} x_{c}}\right)=\operatorname{Real}\left(\frac{l_{3}+l_{2} y_{c}}{-l_{1} y_{c}}\right) \\
\beta=\left|\operatorname{Imag}\left(\frac{-l_{2} x_{c}}{l_{3}+l_{1} x_{c}}\right)\right|=\left|\operatorname{Imag}\left(\frac{l_{3}+l_{2} y_{c}}{-l_{1} y_{c}}\right)\right|
\end{array}\right.
$$

According to the previous equation, the metric rectification parameters can be calculated as long as at least one coordinate $-x_{c}$ or $y_{c}-$ of the ICPs is known. Parameter $\beta$ must always be positive [9] since a negative value mirror-flips the rectified plane.

\section{References}

1. G. Lippmann, "La photographie integràle," Comptes-Rendus Academie des Sciences 146, 446-451 (1908).

2. J.-Y. Son and B. Javidi, "Three-dimensional imaging methods based on multiview images," J. Display Technol. 1, 125 (2005).

3. J.-H. Park, Y. Kim, J. Kim, S.-W. Min, and B. Lee, "Threedimensional display scheme based on integral imaging with three-dimensional information processing," Opt. Express 12, 6020-6032 (2004).

4. J.-S. Jang and B. Javidi, "Formation of orthoscopic threedimensional real images in direct pickup one-step integral imaging," Opt. Eng. 42, 1869-1870 (2003)

5. Y.-T. Lim, J.-H. Park, K.-C. Kwon, and N. Kim, "Resolutionenhanced integral imaging microscopy that uses lens array shifting," Opt. Express 17, 19253-19263 (2009).

6. G. Passalis, N. Sgouros, S. Athineos, and T. Theoharis, "Enhanced reconstruction of three-dimensional shape and texture from integral photography images," Appl. Opt. 46, 5311-5320 (2007).

7. J.-H. Park, K. Hong, and B. Lee, "Recent progress in threedimensional information processing based on integral imaging," Appl. Opt. 48, H77-H94 (2009).

8. N. Sgouros, I. Kontaxakis, and M. Sangriotis, "Effect of different traversal schemes in integral image coding," Appl. Opt. 47, D28-D37 (2008).
9. D. Liebowitz and A. Zisserman, "Metric rectification for perspective images of planes," in Proceedings of the 1998 IEEE Computer Society Conference on Computer Vision and Pattern Recognition (Cat. No. 98CB36231) (IEEE, 1998), pp. 482-488.

10. K. Hong, J. Hong, J.-H. Jung, J.-H. Park, and B. Lee, "Rectification of elemental image set and extraction of lens lattice by projective image transformation in integral imaging," Opt. Express 18, 12002-12016 (2010).

11. E. T. Koufogiannis, N. P. Sgouros, and M. S. Sangriotis, "Robust integral image rectification framework using perspective transformation supported by statistical line segment clustering," Appl. Opt. 50, H265-H277 (2011).

12. E. T. Koufogiannis, N. P. Sgouros, and M. S. Sangriotis, "Perspective rectification of integral images produced using hexagonal lens arrays," in IIH-MSP 2012: The Eighth International Conference on Intelligent Information Hiding and Multimedia Signal Processing (IEEE, 2012), pp. 75-78.

13. R. I. Hartley and A. Zisserman, Multiple View Geometry in Computer Vision, 2nd ed. (Cambridge University, 2004).

14. H. Ip and Y. Chen, "Planar rectification by solving the intersection of two circles under 2D homography," Pattern Recogn. 38, 1117-1120 (2005).

15. M. Lourakis, "Plane metric rectification from a single view of multiple coplanar circles," in Proceedings of the International Conference on Image Processing (ICIP) (IEEE, 2009), pp. 509-512.

16. N. Otsu, "A threshold selection method from gray-level histograms," IEEE Trans. Syst. Man Cybern. 9, 62-66 (1979).

17. R. C. Gonzalez and R. E. Woods, Digital Image Processing, 2nd ed. (Addison-Wesley, 2001).

18. G. Bradski, "The OpenCV Library," Dr. Dobb's J. Software Tools 25 120, 122-125 (2000).

19. P. Kovesi, "MATLAB and octave functions for computer vision and image processing," http://www.csse.uwa.edu.au/ pk/ Research/MatlabFns/index.html.

20. S. S. Athineos, N. P. Sgouros, P. G. Papageorgas, D. E. Maroulis, M. S. Sangriotis, and N. G. Theofanous, "Photorealistic integral photography using a ray-traced model of capturing optics," J. Electron. Imaging 15, 043007 (2006). 